\title{
Planificación Estratégica: antecedentes de aplicación y su vigencia en un mundo contemporáneo.
}

\begin{abstract}
Strategic Planning: application background and its relevance in a contemporary world.
\end{abstract}

Jenny Lilian Basantes Ávalos. ${ }^{1}$, Eduardo Xavier Centeno Parra. ${ }^{2}$, Ernesto Marcelo Bonilla Torres. ${ }^{3} \&$ Rene Abdón Basantes Avalos. ${ }^{4}$

Recibido: 18-03-2021 / Revisado: 25-03-2021 /Aceptado: 15-04-2021/ Publicado: 05-05-2021

\begin{abstract}
DOI: https://doi.org/10.33262/concienciadigital.v4i2.1.1741

The use of strategy and planning dates back to the dawn of mankind, the first inhabitants relied on it for the development of their first tasks; activities related almost entirely to war but managed to develop this practice over the years. Already in modern times, planning became the foundation of business success of the companies of the twentieth century; however, with the passing of time, the development of this administrative tool has not only not lost its validity, but on the contrary, in a highly complex and competitive world, it is necessary to take firm and safe steps that enable an adequate use of resources and promote the fulfillment of organizational objectives. The present study sought to find the relationship between the theory of strategic planning and its support in the results of Ecuadorian companies that have been recognized for their work, and that in their official means of communication state that they use strategic planning as the basis of their

${ }^{1}$ Escuela Superior Politécnica de Chimborazo; Facultad de Administración de Empresas. Riobamba, Ecuador. jenny.basantes@espoch.edu.ec; https://orcid.org/0000-0002-1604-3510

${ }^{2}$ Escuela Superior Politécnica de Chimborazo; Dirección de Evaluación y Aseguramiento de la Calidad. Riobamba, Ecuador. xavier.centeno@espoch.edu.ec; https://orcid.org/0000-00034311-7228

${ }^{3}$ Escuela Superior Politécnica de Chimborazo, Facultad de Administración de Empresas. Riobamba, Ecuador. ernesto.bonilla@espoch.edu.ec; https://orcid.org/0000-0003-3539-1614

${ }^{4}$ Universidad Nacional de Chimborazo, Facultad de Ciencias Políticas y Administrativas. Riobamba, Ecuador. rbasantes@unach.edu.ec; https://orcid.org/0000-0002-7166-5427.
\end{abstract}


success. An exhaustive bibliographic review made it evident that despite the years, the formulation of planning and its adequate implementation guide the achievement of organizational objectives.

Keywords: Management, Business Management, Strategic Planning, Organizational Success

\section{Resumen}

El empleo de la estrategia y la planificación data de los albores de la humanidad, los primeros habitantes confiaron en ella para el desarrollo de sus primeras tareas; actividades relacionadas casi en su totalidad con la guerra pero que logró desarrollar esta práctica a lo largo de los años. Ya en tiempos modernos la planeación se convirtió en el fundamento del éxito empresarial de las compañías del sigo XX; sin embargo y con el pasar del tiempo el desarrollo de esta herramienta administrativa no solo que no ha perdido vigencia; sino que por el contrario, en un mundo altamente complejo y competitivo es necesario dar pasos firmes y seguros que posibiliten un uso adecuado de los recursos y promuevan el cumplimiento de objetivos organizacionales. El presente estudio buscó encontrar la relación entre la teoría de la planificación estratégica y su respaldo en resultados de empresas ecuatorianas que han sido reconocidas por su labor, y que en sus medios de comunicación oficiales manifiestan emplear la planificación y estratégicas como base de su éxito. Una exhaustiva revisión bibliográfica logró evidenciar que a pesar de los años la formulación de la planificación y su adecuada implementación guían al acometimiento de los objetivos organizacionales.

Palabras claves: Administración, Gestión Empresarial, Planificación estratégica, Éxito organizacional.

\section{Introducción}

El concepto de Planificación Estratégica ha ido evolucionando con el pasar del tiempo y utilizado por las antiguas civilizaciones sin una perspectiva empresarial sino más bien como herramienta para crear tácticas de guerra. (Villacís Herrera, A., Barreiros Cabrera, Adriana Elizabeth, 2012). La planificación estratégica como disciplina nació y se desarrolló en el sector privado Estado Unidense en la década de 1960, su máximo apogeo como modelo de gestión se dio en la década de 1970, para decaer en la década de 1980, debido a la complejidad ambiental que comenzaron a experimentar las organizaciones estado unidenses, empero esta herramienta no ha perdido por completo su vigencia, pues es utilizada como mecanismo orientador del orden organizacional. (Ayuzabet de la Rosa Alburquerque, Oscar Lozano Carrillo, 2019).

García, J. (2015) al respecto nos dice que "La planificación estratégica es una herramienta que permite a las organizaciones prepararse para enfrentar las situaciones que se presentan en el futuro, ayudando con ello a orientar sus esfuerzos hacia metas realistas de 
desempeño, por lo cual es necesario conocer y aplicar los elementos que intervienen en el proceso de planificación" (García, J., 2015, p. 9).

En la actualidad, es fundamental que las organizaciones implementen la planificación estratégica, ya que muchos empresarios deciden iniciar una organización, pero pocos son los que logran sostenerlas con éxito en el tiempo y aumentar el valor de la misma. (Fernández Hurtado, S., Martínez Martínez, L., \& Ngono Fouda, R., 2019). Esto se debe al desconocimiento de factores fundamentales que se deben tener en cuenta para garantizar el buen funcionamiento de la organización, la implementación de la planeación estratégica evitará un panorama desfavorable de incertidumbre en los objetivos y metas.

$\mathrm{Al}$ momento de crear un negocio es necesario enfocarse en realizar un plan estratégico de las actividades a realizarse para la creación de la misma, este plan tiene que ser claro y conciso ya que ayudará a cumplir de mejor manera lo propuesto y así obtener resultados favorables para la organización, ayudará a minimizar costos y tiempo, entre otras ventajas.

La planificación estratégica se encuentra alineada al eje de desarrollo económico que tiene como objetivo generar condiciones favorables para el desarrollo de alternativas productivas basado en el aprovechamiento del potencial productivo, hídrico, forestal, turístico y comercial; el fortalecimiento empresarial a través de esquemas de financiación que fomenten la generación de valor agregado y el crecimiento de la productividad, las oportunidades de empleo y la generación de ingresos, impulso de las capacidades de las personas, y la capacidad de pensar y actuar en lo global como en lo local. (Gutierrez, 2019).

Además, la planificación estratégica debe ser vista como un proceso en el cual se toma en cuenta el porvenir, las decisiones actuales, la definición de metas, objetivos, políticas y estrategias para desarrollar planes a mediano y largo plazo, esto es lo que permite ver a futuro y adelantarse a los posibles problemas. (Salazar, Dalmary \& Romero, Gerardo Enrique, 2006)

Su desarrollo constituye un proceso gerencial que desplaza el énfasis en el ¿qué lograr? (objetivos) al ¿qué hacer? (estrategias) y se concentra solo en aquellos objetivos factibles de lograr y en qué áreas competir, en correspondencia con las oportunidades y amenazas que ofrece el entorno. Este proceso consiste en responder: ¿A dónde queremos ir?, ¿Dónde estamos hoy?, ¿A dónde debemos ir?, ¿A dónde podemos ir?, ¿A dónde iremos?, ¿Cómo estamos llegando a nuestras metas? - Y está formada por seis componentes fundamentales: los estrategas, el direccionamiento estratégico, el diagnóstico estratégico, las opciones estratégicas, formulación estratégica y auditoría estratégica. (Leal de Valor, D.Y., Bolívar de Muñoz, M.E., \& Castillo Torrealba, C.O., 2011)

La primera cuestión es entender la planificación como un sistema compuesto por un grupo de componentes, cada uno de los cuales tiene una función que cumplir y un concepto asociado, si nos acercamos al asunto desde la perspectiva de la dirección por objetivos. (Concepción Báez, Carlos M, 2007) Podrían definirse tres elementos básicos: los 
objetivos, los criterios de medida y las acciones, el objetivo es el propósito, lo que se desea y parece probable obtener en el período de tiempo que se planifica; el criterio de medida es el indicador (Cañedo Rubén, 1997), la forma en que se medirá que el objetivo se ha cumplido, y las acciones son las actividades que deben desarrollarse para garantizar el criterio de medida, es decir, el indicador que nos hemos propuesto.

Uno de los principales aportes se presenta cuando las empresas, al contar con una planificación estratégica bien elaborada genera beneficios tangibles alineados a los objetivos establecidos; entre los cuales se puede destacar: la proactividad, adaptación al cambio, la creación de equipos de trabajo eficientes y la toma de decisiones eficaz (Gómez Ortiz \& Rosa Amalia, 2008). En cuanto a los lineamientos de desarrollo del País la planeación estratégica tiene relación con el objetivo N. 5 del Plan Nacional del Buen Vivir, que menciona "Impulsar la productividad y competitividad para el crecimiento económico sostenible de manera redistributiva y solidaria”. (Gutierrez, 2019).

Sobre su contribución a la alta dirección, orienta a los empresarios y los ayuda a enfocarse en el futuro y en el presente; de esa manera se refuerza los principios adquiridos en la Visión y Misión, priorizando en el destino de los recursos, mejorando el desempeño de la Organización todo esto nos lleva de manera efectiva el rumbo de la Organización (Román Muñoz, 2010), facilitando la acción innovadora de dirección y liderazgo. Permite enfrentar los principales problemas de la Organización, enfrentar el cambio en el entorno y develar las Oportunidades y las Amenazas.

El desarrollo de la Planificación Estratégica genera beneficios adicionales y relacionados con la capacidad de realizar una gestión eficiente, liberando recursos humanos, financieros, tecnológicos y materiales, lo que ayuda en la eficiencia productiva y en una mejor calidad de vida y de trabajo para todos los miembros de la organización y lo más importante se centra en los procesos de fabricación de dicha organización ya que ayuda a mejorar los niveles de Productividad, conducentes al logro de obtener rentabilidad. (Tito Huamaní, P. L, 2003)

Optimiza los procesos en las áreas al traducir los resultados en logros sinérgicos, contribuye a la creación y redefinición de Valores Corporativos, que se traducen en:

- Trabajo en Equipo.

- Compromiso con la Organización.

- Calidad en el Servicio.

- Desarrollo Humano (Personal y Profesional)

- Innovación y Creatividad

- Liderazgo, etc.

Entre los factores que se deben estudiar para la implementación de la planificación estratégica se mencionan los siguientes:

Los factores que influyen en el presente y futuro de las organizaciones son: 
El entorno: La organización existe en el contexto de un complejo mundo comercial, económico, político, tecnológico, cultural y social. Este entorno cambia y es más complejo para unas organizaciones que para otras. Puesto que a la estrategia le incumbe la posición que mantiene una empresa con relación a su entorno, la comprensión de los efectos del entorno en la empresa es de importancia capital para el análisis estratégico. Los efectos históricos y medio-ambientales en la empresa deben estudiarse, así como los efectos presentes y los futuros cambios en las variables del entorno. Esta es una de las tareas fundamentales por la amplia gama de variables del entorno. Muchas de estas variables harán aparecer algún tipo de oportunidades, en tanto que otros amenazarán a la organización. (Tito Huamaní, P. L, 2003)

Los recursos: Así como existen influencias externas sobre la organización y sobre su elección de estrategias, también existen influencias internas. Una de las formas de estudiar la capacidad estratégica de una organización es considerar sus puntos fuertes y sus puntos débiles, qué es lo que la organización hace bien y en qué falla, o donde se encuentra en ventaja o desventaja competitiva. Estos puntos fuertes y débiles pueden ser identificados a través del estudio de las áreas, Recursos Humanos y recursos materiales de la organización, como las instalaciones, su estructura financiera y sus productos/servicios (Tito Huamaní, P. L, 2003).

Las expectativas: Los que esperan del futuro los diferentes agentes es importante, pues influirá en lo que se considera aceptable en términos de estrategias anticipadas por la Alta Dirección. No obstante, las creencias y los supuestos que constituyen la Cultura Organizacional, aunque menos explícitas, también tienen una influencia importante. Las influencias del entorno y de los recursos sobre la organización han de interpretarse a través de estas creencias y supuestos. Así, dos grupos de directivos, posiblemente trabajando en diferentes divisiones de una organización, pueden llegar a distintas conclusiones sobre la estrategia, aunque se enfrenten a implicaciones de recursos y de los entornos similares. La influencia que prevalezca, va a depender del grupo que tenga mayor poder, y entender esto será de la mayor importancia para reconocer por qué se sigue una estrategia adoptada. (Tito Huamaní, P. L, 2003)

Las bases: El entorno, los recursos y las expectativas, en el marco cultural y político de la organización proporcionan las bases del. análisis estratégico. Sin embargo, para comprender en que posición estratégica se encuentra una empresa, es necesario considerar también en qué medida la orientación e implicaciones de la estrategia actual y los objetivos que sigue la organización están en línea con las implicaciones que pueden afrontarlas. En este sentido, tal análisis debe realizarse teniendo presentes las perspectivas del futuro, (Tito Huamaní, P. L, 2003)

La estrategia actual capaz de lidiar los cambios que tienen lugar en el entorno de la organización es bastante improbable que exista un perfecto ajuste entre la estrategia actual y la imagen que emerge del análisis estratégico. El grado en que 
haya aquí un desajuste, es el grado del problema estratégico que arrastra la Alta Dirección de la Organización. La fase más importante del Planeamiento Estratégico es la puesta en práctica del mismo. Esto lo logramos mediante el Planeamiento Operativo por parte de cada gerencia o área de trabajo. El objetivo es preparar a cada negocio para hacer frente a diferentes escenarios, con el fin no sólo de anticiparse a los cambios, sino también cómo deben manejarlos o "gerenciarlos", de modo que sean efectivos en el análisis de sensibilidades, desarrollando un marco de trabajo que actúe a la vez como medio de control y equilibrio. (Tito Huamaní, P. L, 2003)

Este estudio busca encontrar evidencia entre la teoría de diversos autores sobre la importancia de la planificación estratégica y los resultados de empresas del medio que han alcanzado reconocimientos y han manifestado el empleo de la planificación y la estrategia como medio para lograrlo.

\section{Metodología}

Con un enfoque cualitativo y un nivel exploratorio, este trabajo se orientó a descubrir o ampliar la información sobre un objeto o materia de estudio específico, en este caso sobre la Planificación Estratégica y su vigencia en la actualidad, a fin de obtener nuevos datos, que se traduzca en nuevos conocimientos, hace referencia al primer acercamiento a un tema específico antes de abordarlo en un trabajo investigativo más profundo en un proceso para tener información básica relacionada con el problema de investigación. (Valducea, M., 2019)

La fuente principal de información fueron trabajos de investigación publicados en revistas científicas con el fin de aproximarnos al tema propuesto. Estos documentos de ata rigurosidad científica permitió a los investigadores profundizar y adquirir mayor entendimiento de los principios, conceptos y experiencias en el tema en cuestión.

La recopilación de información se realizó principalmente en revistas de artículos científicos de alto y mediano impacto de bases como Scielo, Redalyc, entre otras.

Para confirmar las conjeturas obtenidas en la fundamentación teórica de la parte inicial de este documento sobre los resultados que obtiene una empresa que cuenta con una planificación estratégica en aplicación, se analizó la información de las empresas mejor puntuados a nivel nacional; esto datos fueron extraídos del informe de Great Place to Work de su informe denominado Mejores Empresas para Trabajar Ecuador 2020. Sobre esta empresa, el portal especializado en negocios refiere que:

Great Place to Work®, se ha consolidado como la autoridad global en cultura laboral. Durante los últimos 30 años, a nivel mundial ha ayudado a más de 15 mil empresas a prosperar durante la Era del Internet y en los próximos 10 años tiene el firme propósito de ayudar a más de 100 mil empresas a conseguir sus objetivos de negocio en la Era de la Transformación Digital. (Ekos, 2021). 
De este informe, se analizó la información publicada en sus medios de comunicación oficiales por la empresas que resultaron ganadoras en cada categoría.

\section{Discusión y Resultados}

El informe de las Mejores Empresas para Trabajar Ecuador 2020 de Great Place to Work arrojo los siguientes resultados.

Tabla 1: Categoría de hasta $\mathbf{5 0 0}$ colaboradores:

\begin{tabular}{ll}
\hline Posición & \multicolumn{1}{c}{ Empresa } \\
\hline Primera & DHL \\
Segunda & Johnson \& Johnson \\
Tercera & Roche \\
Cuarta & DirecTV \\
Quinta & Nuo
\end{tabular}

Fuente: https://www.ekosnegocios.com/articulo/mejores-empresas-para-trabajar-ecuador-2020.

Tabla 2: Categoría de más de $\mathbf{5 0 0}$ colaboradores:

\begin{tabular}{ll}
\hline Posición & \multicolumn{1}{c}{ Empresa } \\
\hline Primera & Banco de Guayaquil \\
Segunda & LETERAGO del \\
& $\begin{array}{l}\text { Ecuador SA } \\
\text { Tercera }\end{array}$ \\
Naturisa
\end{tabular}

Fuente: https://www.ekosnegocios.com/articulo/mejores-empresas-para-trabajar-ecuador-2020.

En el caso de DHL, empresa ganadora en la primera categoría, manifiesta su estrategia implementada a nivel global hasta el año 2025; la cual se ha establecido de la siguiente manera:

\section{ESTRATEGIA 2025}

Brindamos Excelencia en un Mundo Digital

Deutsche Post DHL Group ha logrado un progreso significativo con la Estrategia 2020. Debido a su extensión geográfica y amplia gama de soluciones logisticas, el Grupo ahora está mejor posicionado que nunca. Con la "Estategia 2025 Entregando excelencia en un mundo digital", la compañía está sentando las bases para continuar su exitosa trayectoria de crecimiento más allá de 2020.

Las cuatro tendencias más importantes que han estado afectando la logística en los últimos años también darán forma a la industria en el futuro: Globalización, Digitalización, E-Comercio y Sustentabilidad. La Estrategia 2025 es la respuesta del Grupo a estos. La compañía se basará en estas tendencias para aprovechar el potencial de crecimiento rentable a largo plazo dentro de sus negocios de logística centrales, al mismo tiempo que intensificará la transformación digital que ya está en marcha en todo el Grupo. 
Todos los esfuerzos se centran en las tres líneas finales establecidas en la Estrategia 2020, que continúan proporcionando la base para la Estrategia 2025. En consecuencia, El Grupo Deusche Post DHL pretende ser reconocido como Empleador, Proveedor, e Inversor de elección en todas sus actividades. (DHL, 2021).

En el caso del Banco de Guayaquil, primer puesto en la categoría de más de 500 colaboradores comunica su planificación y estrategia de sostenibilidad y mantenimiento en el mercado en su portal web; el texto pertinente señala:

Estructuramos nuestros esfuerzos en la Estrategia de Sostenibilidad, construida a partir de los temas materiales y articulada en 5 pilares, integrados a su vez por diferentes líneas de trabajo. Cada línea cuenta a su vez con una selección de iniciativas priorizadas en el tiempo, algunas que ya veníamos desarrollando y otras nuevas con las que nos fortalecemos. Disponemos de un plan de acción para cada iniciativa, incluyendo indicadores de monitoreo. Su implementación es posible gracias a la colaboración de diferentes equipos y áreas del Banco. (Banco de Guayaquil, 2021),

La revisión de la información de las empresas de las categorías presentadas en las tablas 1 y 2 en sus medios de comunicación oficiales dan cuenta de la vigencia de la planificación estratégica; pues todas ellas poseen esta herramienta de alta gerencia y ligan sus resultados al cumplimiento de sus objetivos.

De forma adicional estas compañías se encuentran en el ranking de empresas con mayores ingresos en el país. Pues y como señalan Guzmán et.al. (2001):

Planificar implica tener visión, entendida ésta como la representación mental o imagen -objetivo del futuro deseable y posible. La visión articula la percepción de un futuro realista, creíble y atractivo para un individuo, un grupo una organización, una región un país con lo que ahora existe. Ella debe expresar las aspiraciones fundamentales de los diferentes actores. La visión debe ser alcanzable y debe definir la dirección del proceso de cambio. (Guzmán Saavedra, Castro Zea, Restrepo Quintero, \& Rojas Rojas, 2001)

Se reafirma entonces que el primer paso que se debe dar al momento de poner en marcha una idea de negocio es realizar un plan estratégico para contar con una guía hacia el éxito, no solo para alcanzar sus logros, sino para hacerlo con la mayor celeridad posible, de tal forma que se pueda organizar las actividades y tener un adecuado funcionamiento tanto interno como externo de la empresa.

Entre los aspectos en común empleados por las empresas analizadas se determinó varios puntos en común:

- Definición de objetivos.

- Alcanzar metas ahorrando esfuerzos de tiempo y dinero.

- Incorporación de las partes interesas a la formulación de la estrategia. 
- Aplicación de los conceptos contemporáneos de gestión y planificación.

- Organización y coordinación de ideas y recursos.

- Conocimiento de la empresa, del contexto y la competencia.

- Soporte en las tecnologías de la información.

- Capitación permanente de su talento humano.

\section{Conclusiones}

- Los resultados obtenidos de la presente investigación permitieron identificar que la planificación ha sido históricamente una actividad desarrollada por el ser humano inicialmente para fines militares; sin embargo y con el pasar del tiempo esta fue empleada por los administradores para el establecimiento de metas y objetivos en sus empresas y controlar sus operaciones. También se pudo identificar que como una disciplina de la administración de empresas, la planificación estratégica tuvo su mayor difusión en empresas de mediados del siglo XX. A pesar de su larga historia se pudo evidenciar que organizaciones y empresas exitosas de hoy en día siguen confiando en su utilidad y pertinencia, pues basan sus resultados en estrategias formuladas a partir de la construcción de este documento. Es innegable entonces que si busca alcanzar metas en la empresa, estas deben ser debidamente planificadas, comunicadas y entendidas por todos los colaboradores para lograr los propósitos deseados.

\section{Referencias bibliográficas.}

Ayuzabet de la Rosa Alburquerque, Oscar Lozano Carrillo. (2019). Planeación estratégica y organizaciones públicas: experiencias y aprendizajes a partir de un proceso de intervención. Gestión y Estrategia, 37 (1), 61-77. Recuperado de: http://gestionyestrategia.azc.uam.mx/index.php/rge/issue/view/30.

Banco de Guayaquil (2021). Conócenos. Recuperado de https://www.bancoguayaquil.com/conocenos/sostenibilidad

Cañedo Andalia, Rubén. (1997). Elementos conceptuales útiles para la implementación de los sistemas de calidad. ACIMED, 5(4), 1-10. Recuperado de http://scielo.sld.cu/scielo.php?script=sci_arttext\&pid=S1024-

$94351997000400001 \& \operatorname{lng}=$ es\&tlng=es.

Concepción Báez, Carlos M. (2007). La planificación estratégica como herramienta. ACIMED, 16(2) Recuperado de: http://scielo.sld.cu/scielo.php?script=sci_arttext\&pid=S102494352007000800014\&lng=es\&tlng=es.

DHL (2021). Estrategia. Recuperado de: https://www.dhl.com/ec-es/home/quienessomos/estrategia-2025.html 
Ekos (2021). Mejores Empresas para Trabajar Ecuador 2020. https://www.ekosnegocios.com/articulo/mejores-empresas-para-trabajar-ecuador2020

Fernández Hurtado, S., Martínez Martínez, L., \& Ngono Fouda, R. (2019). Barreras que dificultan la planeación estratégica en las organizaciones. Tendencias, 20(1), 254279. https://doi.org/10.22267/rtend.192001.108.

García, J. (2015). Planeación estratégica. Ccichonduras, 10. Recuperado de: http://www.ccichonduras.org/website/Descargas/presentaciones/2015/04Abril/PLANIFICACION_ESTRATEGICA.pdf

Gómez Ortiz, Rosa Amalia. (2008). El liderazgo empresarial para la innovación tecnológica en las micro, pequeñas y medianas empresas. Pensamiento \& Gestión, (24), 157-194. Recuperado de: http://www.scielo.org.co/scielo.php?script=sci_arttext\&pid=S1657$62762008000100007 \& \operatorname{lng}=\mathrm{en} \& \ln \mathrm{ln}=\mathrm{es}$.

Gutiérrez, C. (2019). Plan estratégico para el desempeño laboral. Repositorio institucional Uniandes.

Guzmán Saavedra, R., Castro Zea, L. E., Restrepo Quintero, O., \& Rojas Rojas, A. (2001). Planificación del Desarrollo. Bogotá: Editora Géminis Ltda.

Jimena, G. (2015). Vp Consultores. Recuperado de: http://www.ccichonduras.org/website/Descargas/presentaciones/2015/04Abril/PLANIFICACION_ESTRATEGICA.pdf

Leal de Valor, D.Y., Bolívar de Muñoz, M.E., \& Castillo Torrealba, C.O.. (2011). La Planificación Estratégica como proceso de integración de un equipo de salud. Enfermería Global, 10(24). Recuperado de: https://dx.doi.org/10.4321/S169561412011000400015

Román Muñoz, Olga (2010). El pensamiento estratégico. Una integración de los sentidos con la razón. Revista Científica Guillermo de Ockham, 8 (2), 23-36. Recuperado de: https://www.redalyc.org/articulo.oa?id=105316833003

Salazar, Dalmary \& Romero, Gerardo Enrique (2006). Planificación. ¿Éxito Gerencial ?. Multiciencias, $\quad 6 \quad(1), \quad 0 . \quad$ Recuperado de: https://www.redalyc.org/articulo.oa?id=90460103.

Tito Huamaní, P. L. (2003). Importancia del Planeamiento Estratégico para el Desarrollo Organizacional. Gestión En El Tercer Milenio, 5(10), 105-110. Recuperado de: https://doi.org/10.15381/gtm.v5i10.9927 administrativas, 6.

Valducea, M. (2019). Metodología de la investigación. Obtenido de https://selinea.unidep.edu.mx/files/528to832_r649220160427120000289061.pdf 
Villacís Herrera, A., Barreiros Cabrera, Adriana Elizabeth (2012). Planificación Estratégica como una herramienta de gestión para promover la competitividad en la Empresa KAWA MOTORS. Recuperado de: http://www.dspace.uce.edu.ec/handle/25000/176.

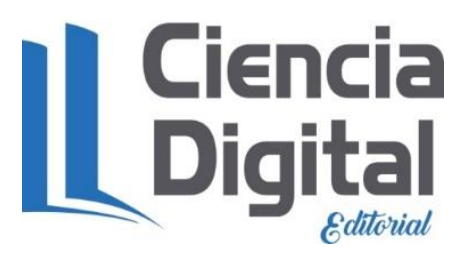




\section{PARA CITAR EL ARTÍCULO INDEXADO.}

Basantes Ávalos, J. L., Centeno Parra, E. X., Bonilla Torres, E. M., \& Basantes Avalos, R. A. (2021). Planificación Estratégica: antecedentes de aplicación y su vigencia en un mundo contemporáneo . ConcienciaDigital, 4(2.1), 154-165. https://doi.org/10.33262/concienciadigital.v4i2.1.1741

\section{¿Ciencia}

El artículo que se publica es de exclusiva responsabilidad de los autores y no necesariamente reflejan el pensamiento de la Revista Conciencia Digital.

El artículo queda en propiedad de la revista y, por tanto, su publicación parcial y/o total en otro medio tiene que ser autorizado por el director de la Revista Conciencia Digital.

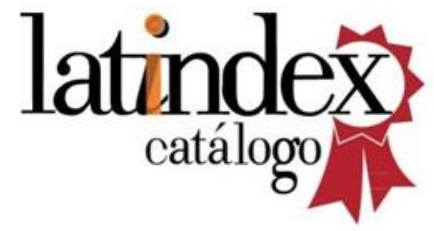

